\title{
ATTITUDE TOWARDS STRESS OF CANCER AND CARDIAC PATIENTS
}

\author{
Md. Azibar Rahman*, Shawkat Ara**, Md. Enamul Haque***, Mohd. Ashik Shahrier**** \\ *Lecturer, Department of Psychology, Shalua Degree College, Rajshahi. \\ ***Ex-Professor, Department of Psychology, University of Rajshahi \\ *** Professor, Department of Psychology, University of Rajshahi. \\ ****Lecturer, Department of Psychology, University of Chittagong*. \\ Corresponding Author, Cell: 01718-183582, Email: ashikpsy@cu.ac.bd
}

\begin{abstract}
The study focused on exploring the attitude towards stress of cancer and cardiac patients as related to gender and SES. The sample of the study comprised of 360 respondents selected purposively. Attitude towards Stress Scale (Rahman, 2010) was used for data collection. The sample was equally divided into three categories-cancer, cardiac and normal on the basis of type of individuals ( $\mathrm{N}=120$ for each category).Again they were equally subdivided into male and female on the basis of gender ( $\mathrm{N}=60$ for each group).Each category was again equally subdivided into lower middle and upper middle on the basis of SES ( $\mathrm{N}=30$ for each group). Results analyzed through ANOVA revealed that the main effects for type of individuals, gender and SES were statistically significant. That is, both cancer and cardiac patients expressed higher stress attitudes as compared to normal individuals, females expressed higher stress attitudes as compared to males and lower middle SES individuals expressed higher stress attitudes as compared to upper middle SES. Again interaction effect of a three-way analysis of variance involving type of individual, gender and SES was statistically significant.
\end{abstract}

Key Words: Attitude, stress, cancer, cardiac, gender, SES

সারাংশ: লিঙ্গ এবং আর্থ-সামাজিক অবস্থা অনুযায়ী ক্যান্সার এবং কার্ডিয়াক রোগে আক্রান্ত ব্যক্তিদের পীড়নের প্রতি মনোভাব জানাই ছিল বর্তমান গবেষণার মুখ্য প্রয়াস। গবেষণার নমুনা হল উদ্দেশ্যমূলকভাবে নির্বাচিত ৩৬০ জন উত্তরদাতা। উপাত্ত সগ্রহের জন্য পীড়নের প্রতি মনোভাব পরিমাপক স্কেল (রহমান, ২০১০) ব্যবহৃত হয়। নমুনাকে ব্যক্তির ধরণের উপর ভিত্তি করে ক্যান্সার আক্রান্ত, কার্ডিয়াক আক্রান্ত এবং স্বাভাবিক ব্যক্তি (প্রতি দলে ১২০ জন করে) এ তিনভাগে ভাগ করা হয়। এরপর তাদেরকে লিঙ্গের ভিত্তিতে পুরুষ এবং মহিলা (প্রতি দলে ৬০ জন করে) এ দু’ভাগে ভাগ করা হয়। প্রত্যেক ক্যাটাগরিকে আবার আর্থ-সামাজিক অবস্থার প্রেক্ষিতে নিম্নমধ্যবিত্ত ও উচ্চমধ্যবিত্ত (প্রতি দলে ৩০ জন করে) এ দু’ভাগে ভাগ করা হয়। ভেদাংক বিশ্লেষণের মাধ্যনে প্রাপ্ত ফলাফল থেকে দেখা যায়, ব্যক্তির ধরণ, লিঙ্গ এবং আর্থ-সামাজিক অবস্থার মুখ্য প্রভাব পরিসংখ্যানিকভাবে তাৎপর্যপূর্ণ হয়েছে। অর্থাৎ,ক্যান্সার এবং কার্ডিয়াক আক্রান্ত রোগীরা স্বাভাবিক ব্যক্তিদের তুলনায় অধিক পীড়নমমূলক মনোভাব প্রকাশ করে, মহিলারা পুরুষদের তুলনায় অধিক পীড়নমূলক মনোভাব প্রকাশ করে, নিম্নমধ্যবিত্তরা উচ্চমধ্যবিত্তদের তুলনায় অধিক পীড়নমূলক মনোভাব প্রকাশ করে। আবার, ব্যক্তির ধরণ, লিঙ্গ ও আর্থ-সামাজিক অবস্থার ত্রি-মুখী পারস্পরিক প্রভাবও পরিসংখ্যানিকভাবে তাৎপর্যপূর্ণ হয়েছে।

\section{Introduction}

Stress is a disruptive physiological and psychological response to events that make us feel threatened or upset our balance in some way. When we sense danger whether it's real or imagined - the body's defenses kick into high gear in a rapid, automatic process known as the "fight-or-flight" reaction, or the stress response. The common manifestations resulting from stress are depression, anxiety, hysteria; cardiovascular accidents, muscular aches and pains, insomnia, gastrointestinal ulcers, hypertension, cancers, sexual dysfunctions, allergies and other mental disorders. A certain amount of stress is an inevitable part of life; as stress researcher Hans Selye (1974) put it, "complete freedom from stress is death". But acute stress can interfere with functioning in every area of our lives and chronic stress has been linked to degeneration of general health, colds and flue, allergies, high blood pressure, increased risk of heart disease, fatal heart attacks and cancer. In reaction to stress, heart rate and blood pressure rise, and the person breathes more rapidly, which allows the lungs to take in more oxygen. Blood flow to the muscles, lungs, and brain may increase by $300-400 \%$.
The spleen releases more blood cells into the circulation, which increases the blood's ability to transport oxygen. The immune system redirects white blood cells to the skin, bone marrow, and lymph nodes; these are areas where injury or infection is most likely. A chronic disease like cancer has an impact on all aspects of a patient's life. Work may be threatened or terminated by the need for extensive treatments or by the debilitating side-effects of the disorder. The patient's psychological state is almost certainly affected in that the diagnosis of a chronic illness can produce extreme fear and anxiety or depression. The term "cancer" refers to more than 100 different diseases, but all involve a malfunction in the mechanisms that control cell reproduction. The normal duplication of cells - a slow, regular process - is replaced by a rapid production of abnormal cells that invade the body's organs and tissues. Some cancers develop quickly; others take decades to become detectable; still others have an irregular and unpredictable course. Several investigators report that people, too, are at increased risk for cancer a year or so after experiencing depression, helplessness, or bereavement. For example, cancer occurs more often than usual among those 
widowed, divorced, or separated. Recent research indicates that the body's release of stress hormones can affect cancer cell functions directly (Reiche et al. 2004; Thaker et al. 2006). Chronic repressed emotions and chronic stress can reduce immune function, making a person more susceptible to diseases, including cancer. In general, stronger relationships have been found between psychological factors and cancer growth and spread than between physiological factors and cancer development (Antoni et al. 2006). Cancer is, as we know, a life changing experience for the cancer sufferer, their friends and families. Cancer and cancer treatment both have rather drastic psychological and physiological effects on the sufferer.

Stress also has a number of negative effects on the heart and circulatory system. Sudden stress increases heart rate, but also causes the arteries to narrow, which may block the flow of blood to the heart. The emotional effects of stress can alter the rhythm of the heart. In addition, stress causes the release of extra clotting factors into the blood, which increases the risk of a clot forming and blocking an artery. Stress also triggers the release of fat into the bloodstream, which temporarily raises blood cholesterol levels. Lastly, it is thought that people who regularly have sudden increases in blood pressure due to mental stress may over time suffer injuries to the inner lining of their blood vessels.Studies of psychiatric patients, community samples, and patients with known heart disease demonstrated that depressive disorders, stressful life events, and poor social support are associated with increased incidence, morbidity, and mortality of atherosclerotic heart disease (Sher, 1998; Tennant, 1999). Considerable evidence suggests that depression is an independent risk factor in the pathophysiologic progression of cardiovascular disease. More and more evidence suggests a relationship between the risk of cardiovascular disease and environmental and psychosocial factors. These factors include job strain, social isolation and personality traits. Two main cardiac disorders, coronary heart disease and hypertension are the largest killers in the world society today. Every year, millions of people die from cardiac degeneration including hypertension, heart failure, stroke and kidney diseases. CHD has been linked to hypertension, diabetes, smoking, obesity, high levels of cholesterol, and low levels of physical activity (American Heart Association, 1984). The least stressful had the lowest prevalence of coronary heart disease, and the most stressful had the highest rate of coronary heart disease. A lack of balance between demands (e.g. job stress) and buffering resource (e.g. social network) evokes negative emotions (such as hopelessness). Negative emotions may lead to smoking, lack of exercise and poor diet, which may in part be reflected by obesity and poor lipid and haemostatic profiles. Hostility may be one of the psychosocial stressors changing physiological mechanisms resulting in heart diseases. Instead, both depression and CHD seemed to develop in Type A's who often give vent to their anger, hostility, and aggression.

A review of the available literature corresponding to the attitudes towards stress of cancer and cardiac patients in relation to gender and SES has been put forward in this section.

Byrnes et al. (1998) proposed a causal model for the association between stress, depression, and cancer. Stress and depression are associated with a deregulation of inflammatory cytokines; stress is associated with increased expression of interleukin (IL)-1b and down-regulation of IL-2, interferon (IFN)-gamma (Interferon), NKA, and major histo-compatibility complex (MHC) class II molecules (Dahlquist et al. 1996). Stress and depression can foster tumor progression by inhibiting expression of MHC class I and II molecules and through NKA reduction. Goldenberg, Libov and colleagues (2002) have found that higher family income and high SES background is associated with higher levels of PTS symptoms in cancer patients. Bennett et al. (2004) found that cancer patients with higher levels of optimism have lower levels of both anxiety and depression. The sex of the parent is assumed to predict levels of distress.2-3 months after cancer diagnosis the mean level of state anxiety has been found to be significantly higher among mothers than fathers (Allen et al. 1997; Dahlquist et al.1996). Esterling et al. (1996) found evidence that social support may modulate the effect of chronic stress on immune function. Social support may be a key moderator of the effect of psychosocial stress on cancer development. In another study, Agnes (1980) studied hostility patterns and life stress in cancer patients. The analysis revealed that females had higher scores on content hostility as compared to normal subjects. Another relevant finding was that the cancer group with low SES reported significantly higher stressful life events as compared to the cancer group of high SES and normal group of low SES. A study by Spiegel (1997) indicate that clear and open communication, expression of appropriate emotion, and collaborative planning and problem-solving enhance the adjustment of high SES breast cancer patients. Conversely, influences that isolate lower middle SES breast cancer patients from others or undermine support can have adverse medical and psychological consequences.

In case of cardiac patients, Roseman and Others (1975) in a prospective study reported that Type A men have 
twice the incidence of coronary heart disease as have type B men. In fact the two-fold increase in CHD risk reported for type $\mathrm{A}$ men was almost exactly replicated for type A women. The CHD Type A association among males in this study was limited to those of white collar socio-economic status (Haynes and others, 1980). A number of studies (Thom et al. 2006; Loomba \& Arora, 2008; Mosca et al. 2007) indicated that heart disease is an important contributor to morbidity and mortality among women. While many of the underlying cardiovascular risk factors are similar for both women and men, cardiovascular disease among women has some unique features. These include a higher frequency of women with sudden death without previous symptoms and an increased mortality among older women compared to men following a myocardial infarction. These factors may contribute to higher coronary heart disease mortality in women compared to men (Mosca et al. 2005). Loomba \& Arora (2008) found that women have a higher mortality rate after an initial myocardial infarction than men, and the majority of women suffering from an acute cardiovascular event do not make a full recovery. Further, the vast majority of middle-aged women have at least one risk factor for heart disease. Bittner (2006) mentioned that although cardiovascular disease can, to a large extent, be prevented by attention to established risk factors, many women are unaware that they are at risk. A recent study Pischon et al. (2008) demonstrated a higher relative risk for coronary heart disease related to the commonly occurring metabolic syndrome among women compared to men. Further, the nature of disease manifestations as well as clinical symptoms may differ across gender. Bedinghaus et al. (2001) found that among women, the atherosclerotic process is more distributed in the vascular tree, and symptoms such as fatigue, dyspnea, and shoulder or neck pain are more commonly associated with myocardial infarction than in men, contributing to a greater diagnostic challenge. Harburg et al. (1973) and James and Kleinbaum (1976) mentioned that persons residing in high socio-ecological stress areas and low SES background have more evidence of hypertension and a higher rate of hypertension mortality than do their counterparts who live in low stress areas and high SES background. Shapiro, Goldstein and Jamner (1995) said that anger may function differently in men and in women. Expressing anger is related to increased blood pressure reactivity in men, whereas suppressing anger is linked to higher blood pressure reactivity in women.

\section{Objective of the Study}

The broad objective of the study was to investigate the attitudes towards stress of cancer and cardiac patients in Bangladesh as related to gender and socio economic status.

\section{Rationale of the study}

This is a comparative study between cancer-normal individuals, cardiac-normal individuals, cancer -cardiac patients. Hence, it is hope that the findings would provide insight understanding the differential coping attitudes toward differential stresses according to the type of individuals. Another importance of the study is to identify some factors of stress of cancer and cardiac patients which would help subsequent sufferers suffering from chronic illnesses to cope with the negative life events and encouraged them to face crucial moment of life in a positive manner. The most important feature of the study is that it is useful in the evaluation and guidance of the phenomena of cancer and cardiac patients in terms of socio-economic condition, gender differences of patients and normal individuals. Lastly, this study would remove confusion and contradiction between patients and normal individuals that may be developed on attitude towards stress in relation to gender and SES differences in Bangladesh. Considered on these perspectives, this study undoubtedly covers a very significant area of research in varied psychosocial aspects of Bangladesh.

\section{Hypotheses of the Study}

$\mathbf{H}_{1}$ : Both cancer and cardiac patients would express higher stress attitudes as compared to normal individuals.

$\mathbf{H}_{2}$ : Females would express higher stress attitudes as compared to males.

$\mathbf{H}_{3}$ : Lower middle SES individuals would express higher stress attitudes as compared to upper middle SES individuals.

\section{Materials and Methods}

Sample: A total of 360 respondents constituted the sample of the study. At first a sheet was set for collecting the personal information of the participants such as name, age, educational qualification, occupation, monthly income, religion, husband/parental income, marital status, health wellbeing, types of illness etc. To identify the participants as cancer or cardiac individuals, a questionnaire consisting of 10 items named 'Cancer-Cardiac Criteria Questionnaire' developed by the researcher was administered in question form through 'yes' or 'no' approach to the respondents. Through this questionnaire individuals were diagnosed whether they were cancer or cardiac patients or not and other questions of this questionnaire indicated how positively or negatively cancer or cardiac patients took their disease and dealt with it. Individuals who were not suffering any chronic disease or any other illness were diagnosed as normal individuals 
and it was confirmed through the personal information sheet that ensured whether they were suffering from any chronic illness or not Through this procedure and after the application of 'Cancer-Cardiac Criteria Questionnaire' some of the subjects were found as cancer patients, some were cardiac and some individuals were found to be normal individuals. Thus, finally three categories of subjects were selected. From them, 120 Ss were selected purposively as cancer patients, 120 cardiac patients and 120 as normal individuals. Then, the subjects were subdivided into males and females comprising 60 participants for each group. Again, these participants were divided into two groups' i.e. upper middle SES and lower middle SES comprising 30 participants for each group. From the three SES related questions of personal information sheet, the SES level of the respondents was determined. These SES related questions consisted of the respondents' education level, occupation and monthly income. Through these three questions, the researcher with his own setup criteria for SES based on respondents' education level, occupation and monthly income by considering the present socioeconomic condition of Bangladesh identified subjects into two categories such as upper-middle and lower middle SES. Thus, the total subjects comprised of 360 respondents selected purposively for this study and after that the main instrument 'Attitude towards Stress Scale' was administered on the sample to know their attitude towards stress.

\section{Instruments}

The following measures were used to collect data of the present study: (1) Personal information sheet (PIS), (2) Cancer-Cardiac Criteria Questionnaire (CCCQ), (3)Attitude Towards Stress (ATS) Scale.

Personal Information Sheet (PIS): A questionnaire was set for collecting the personal information of the subjects, such as name, age, educational qualification, occupation, monthly income, religion, husband/parental income, marital status, health wellbeing, types of illness, SES etc.

\section{Attitude towards Stress (ATS) Scale}

In this study Attitude towards Stress (ATS) Scale constructed by Rahman, A. (2010) was used for collection of data. This scale contains 24 items divided into 6 dimensions, four items for each dimension. These dimensions were psychological stress, situational stress, physiological stress, financial stress, environmental stress and surgical stress. The split half reliability was computed with odds and even numbers of those 24 items scores and the Pearson's ' $r$ ' was found 0.85 . To find out the validity of the scale, correlations between the total
ATS scores with the scores of each dimension were computed which ranged from 0.46 to 0.82 .

\section{Procedure of Data Collection}

In this study, the investigator utilized two measures i.e. PIS and ATS scale to each of the 120 subjects separately for cancer patients, cardiac patients and normal individuals. The investigation was conducted on a sample of three categories - one, cancer patients, two, cardiac patients and three normal individuals. Data of all patients were collected from different medical college hospitals \& health institutes of Rajshahi and Dhaka cities. Data of cancer patients were collected from Rajshahi Medical College Hospital; Rajshahi Cancer Hospital and Research Centre; Dhaka Ahsania Mission Cancer Hospital; Mudabbir Cancer Care Centre, Dhaka; National Cancer Institute Mohakali, Dhaka; Square Hospital, Dhaka; Appollo Hospital, Dhaka; Central Hospital, Dhaka; Delta Hospital, Dhaka. Data of Cardiac patients were collected from Rajshahi Medical College Hospital, Rajshahi Heart Foundation, Dhaka National Cardiac Hospital, Dhaka Ibrahim Cardiac Hospital \& Research Institute; data of normal individuals were collected from different parts of Rajshahi and Dhaka cities. The participants took half an hour to fill up ATS questionnaire. The investigator was able to establish rapport with the participants. They were told that these would help them cope with stress. Thus, they spontaneously responded to all the questions.

\section{Scoring Procedure}

The data of the present study were collected through ATC scale. This scale contains 24 items divided into 6 dimensions, four items for each dimension. There are five alternative responses to each item. These are: (i) strongly agree, (ii) agree (iii) neutral, (iv) disagree and (v) strongly disagree. The responses to various positive items were scored in such a way that 5, 4, 3, 2 \& 1 was respectively given for the five above mentioned alternatives. The responses to various negative items were scored in such a way that $1,2,3,4$ \& 5 was respectively given for the five above mentioned alternatives. Then the total score of each respondent is obtained by adding the scores of all 24 items. Thus for 24 items, the score ranged from 24 to 120 . Thus the highest score indicated highest coping attitudes and the lowest score indicated lowest stress attitudes. Hence, the midpoint was

$=\frac{\text { Highest score }+ \text { Lowest score }}{2}=\frac{120+24}{2}=72$

The scores above this mid point were indicative of higher stress attitudes and the scores under this mid point were indicative of lower stress attitudes. 


\section{Design of the Study}

This is a study of persons with the disease (cancer and cardiac) of interest and a suitable control group of persons without the disease i.e. normal individuals (comparison group, reference group). The potential relationship of a suspected risk factor to the disease i.e. attitude towards stress is examined by comparing the diseased (cancer or cardiac) and non diseased (normal) subjects with regard to how frequently the factor is present in each of the groups (diseased and non diseased) based on certain demographics like gender and SES. Thus, case control design was used for the present study.

\section{Results}

The study used three independent factors such as type of individuals, gender and SES. Type of individuals was divided into cancer patient, cardiac patient and normal individual. Gender was divided into male and female. SES included upper middle SES and lower middle SES. These independent factors were indicative to show differential attitude towards stress. The data of the present study were analyzed through Analysis of Variance (ANOVA).

Table 1 Showing summary of ANOVA involving Type of Individual, Gender and SES on the scores of Attitude towards Stress Scale.

\begin{tabular}{|c|c|c|c|c|}
\hline Source of Variance & Sum of Squares & df & Mean Square & $\mathrm{F}$ \\
\hline Type of Individual (A) & 12764.96 & 2 & 6382.48 & $23.02 * *$ \\
\hline Gender (B) & 12460.90 & 1 & 12460.90 & $44.95^{* *}$ \\
\hline SES (C) & 2340.90 & 1 & 2340.90 & $8.44^{* *}$ \\
\hline $\mathrm{AB}$ & 390.07 & 2 & 195.03 & 0.70 \\
\hline $\mathrm{AC}$ & 511.80 & 2 & 255.90 & 0.92 \\
\hline $\mathrm{BC}$ & 24.54 & 1 & 24.54 & 0.09 \\
\hline $\mathrm{ABC}$ & 2062.96 & 2 & 1031.48 & $3.72 *$ \\
\hline Within Cell (Error) & 96480.53 & 348 & 277.24 & \\
\hline Total & 127036.66 & 359 & & \\
\hline
\end{tabular}

$\mathrm{P}<0.05^{*}, \quad \mathrm{P}<0.01^{* *}$

The results reported in table- 1 showed that main effects for type of individuals $(F=23.02, d f=1 / 348, p<0.01)$, gender $(\mathrm{F}=44.95, \mathrm{df}=1 / 348, \mathrm{p}<0.01)$ and SES $(\mathrm{F}=8.44, \mathrm{df}=1 / 348, \mathrm{p}<0.01)$ were statistically significant. It was also found that interaction effect of a three-way analysis of variance involving type of individual, gender and SES was statistically significant $(\mathrm{F}=3.72, \mathrm{df}=1 / 348, \mathrm{p}<0.05)$.

Table 2 Showing overall mean scores and significant mean differences for the main effect of type of individual on the scores of Attitude towards Stress Scale (N=120 for each group).

\begin{tabular}{l|r|}
\hline \multicolumn{1}{|c|}{ Group } & \multicolumn{1}{c|}{ Mean Scores } \\
\hline Cancer Patients & 81.53 \\
Cardiac Patients & 77.58 \\
Normal Individuals & 67.43 \\
\hline
\end{tabular}

Note: Mean differences were computed using Newman-Keuls formula. $\mathrm{P}<0.01$

Table 3 Showing overall mean scores and significant mean difference for the main effect of gender on the scores of Attitude towards Stress Scale (N=180 for each group).

\begin{tabular}{|l|l|}
\hline Gender & Mean Scores \\
\hline Male & 69.68 \\
Female & 81.36 \\
\hline
\end{tabular}

Table 4 Showing overall mean scores and significant mean difference for the main effect of SES on the scores of Attitude towards Stress Scale (N=180 for each group).

\begin{tabular}{|l|l|}
\hline SES & Mean Scores \\
\hline Upper Middle SES & 72.92 \\
Lower Middle SES & 78.11 \\
\hline
\end{tabular}


Table 5 Showing overall cell means and their significant mean differences of three-way interaction involving type of individual, gender and SES on the scores of Attitude towards Stress Scale ( $\mathrm{N}=30$ for each group).

\begin{tabular}{|c|c|c|c|}
\hline \multirow{2}{*}{ Type of Individuals } & \multirow{2}{*}{ Gender } & \multicolumn{2}{|c|}{ SES } \\
\hline & & Upper Middle SES & Lower Middle SES \\
\hline \multirow[t]{2}{*}{ Cancer Patients } & Male & $75.93_{\mathrm{a}}$ & $78.07 \mathrm{a}$ \\
\hline & Female & $78.93_{\mathrm{a}}$ & $93.20_{b}$ \\
\hline \multirow{2}{*}{ Cardiac Patients } & Male & $66.73_{\mathrm{c}}$ & $76.60_{\mathrm{a}}$ \\
\hline & Female & $83.47_{\mathrm{d}}$ & $83.53 \mathrm{~d}$ \\
\hline \multirow{2}{*}{ Normal Individuals } & Male & $57.93 \mathrm{e}$ & $62.80_{\mathrm{f}}$ \\
\hline & Female & $74.53_{\mathrm{a}}$ & $74.47_{\mathrm{a}}$ \\
\hline
\end{tabular}

Note: Common subscripts do not differ significantly. Mean differences were computed using Newman-Keuls formula (Winer, 1971; PP. 191-195). $\mathrm{P}<0.01$

The interaction effect has been graphically plotted in Figure -1 .

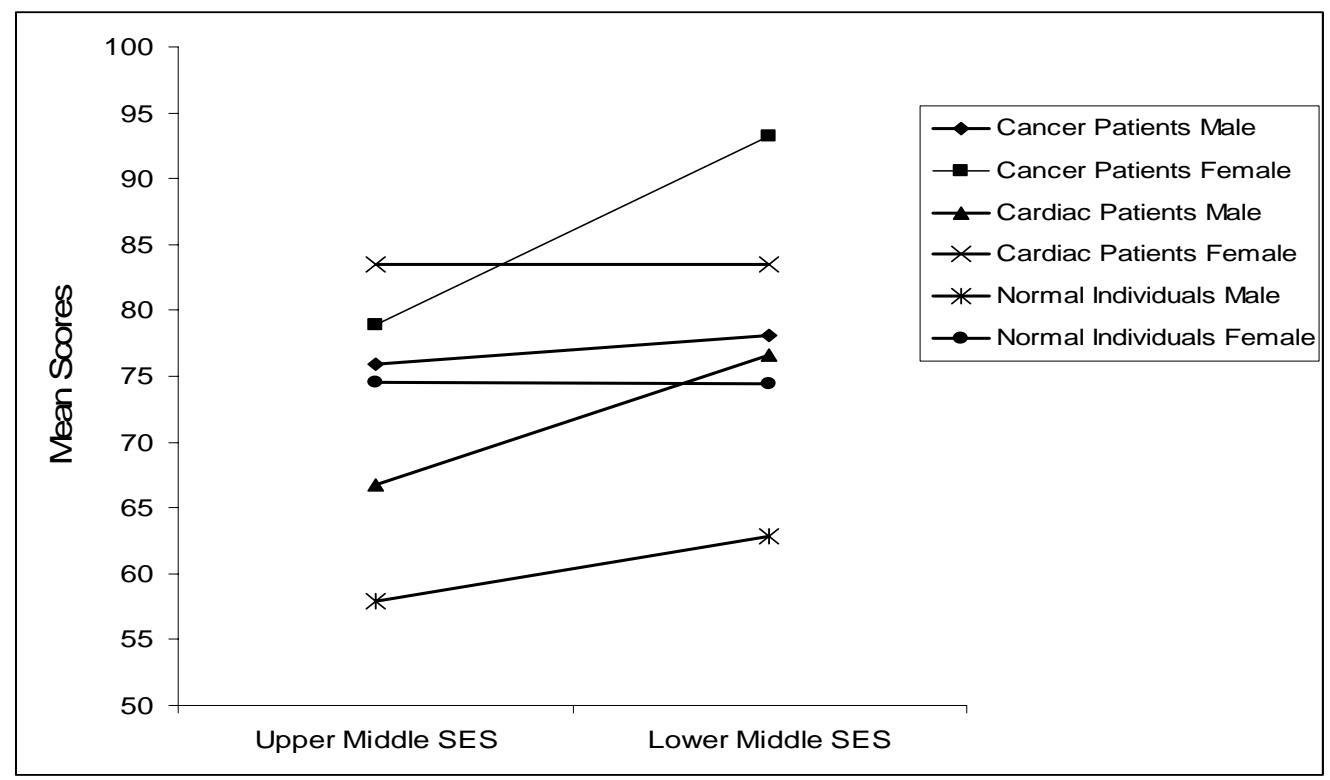

Figure 1 Showing three-way interaction effect between type of individual, gender and SES.

\section{Discussion}

Physiological symptoms of stress are those of arousal; increases in pulse rate, blood pressure, respiration and the level of certain hormones. The emotional symptoms include fear, anxiety and excitement, anger, embarrassment and depression. The cognitive symptoms include obsessive thoughts and an inability to concentrate. The first hypothesis of the present study was that both cancer and cardiac patients would express higher stress attitudes as compared to normal individuals. The findings of the present study have provided empirical confirmation to this hypothesis. The results (Table-1) reported that regardless of gender and SES, cancer patients expressed significantly higher stress attitude cardiac patients and least by normal individuals (Table-2). These findings may be supported from various theoretical imports as well as empirical studies. Several investigators reported that people are at increased risk for cancer a year or so after experiencing depression, helplessness or bereavement. Cancer occurs more often than usual among those widowed, divorced or separated. Stress like depression, anxiety, frustration affects the growth of cancer cells by weakening the body's natural defense against a few proliferating malignant cells (Kessler et al. 1991). After being diagnosed as a cancer patient, the daily activities or work of a patients may de threatened or terminated by the need for extensive treatments or by the debilitating side effects of the disorder. Physical changes that occur, 
loss of income due to job restriction or the need for help from others may lead individuals to feel inferior and it can produce acute stress within his psycho-physical systems (Reiche et al. 2004). Due to problems in social communication and social support, cancer patients are sometimes inadvertently victimized by their family members and friends.

Though the stress patterns of cancer and cardiac patients are similar in most of the cases but some discrepancies remain in the stress pattern of cardiac patients. Two main cardiac disorders, coronary heart disease and hypertension create acute stress among cardiac patients in their daily living activities. While identifying the behavioural and emotional pattern of stress, Rosenman \& Others (1975) reported that type A men have twice the incidence of coronary heart disease as have type-B people. They found that both depression and CHD seemed to develop in type A's who often give vent to their anger, hostility and aggression. Due to these behavioural and emotional stress patterns typeA's have a greater incidence of developing CHD than type B's. Schnall \& others (1990) found that subjects with high occupational stress and difficult work environments faced three times greater risk of having high blood pressure and coronary heart disease than those without occupational stress. Normal individuals in their daily living activities also face different types of stress and in different intensities as much as cancer and cardiac patients. But their responses to these stressful events are somewhat different from those of the cancer or cardiac patients. Life's most stressful events like loss of a loved one, marital separation, death of a spouse, lose of job, man made and natural disasters etc. and less stressful events like daily hassles, noise pollution, air pollution, traffic jam etc. are also faced by normal individuals. But when exposed to these stressful situations, their bodies respond with strong parasympathetic nervous system activity that it helps them to remain quiet and calm in stressful situations. On the contrary, when cancer and cardiac patients are exposed to stressful events, their bodies respond with exceptionally high levels of sympathetic nervous system activity, which causes were and tear on the heart and helps in the rapid production of abnormal cells that invade the body's organs and tissues (Williams et al. 1982; Lazarus, 1980). From the research findings related with the stress patterns of cancer and cardiac patients, it may be said that both cancer and cardiac patients have a pronounced tendency to show higher stress attitude as compared to normal individuals but cancer patients are more likely to show higher stress attitudes than cardiac patients in most of the dimensions of attitudes towards stress.
The second hypothesis of the present study was that female patients would express higher stress attitude as compared to male patients. The findings of the present study have provided empirical confirmation to this hypothesis. The results (Table-1) reported that regardless of type of individual and SES, female patients expressed significantly higher stress attitude as compared to their male counterparts (Table-3). This finding may be supported from various theoretical imports as well as empirical studies. Agnes (1980) studied hostility patterns and life stress in cancer patients consisted of 17 male and 18 females. The analysis revealed that cancer patients, particularly females, had higher scores on content hostility as compared to males. Another relevant finding was that the cancer group reported significantly higher stressful life events as compared to the normal group. Srivastava \& Broota (1987) found that among cancer or cardiac patients, females had experienced significantly higher stress emanating from the death of their mothers before the age of 18 years and lack of closeness with the mother as compared to their male counterparts. The findings also imply that among the remote stress variables only maternal deprivation was a predictor to cancer more frequently in women than men. Schnall \& Others (1990) conducted a study on 215 male female workers relating occupational stress to blood pressure elevations. Researchers found that among subjects with high occupational stress, women faced three times greater risk of having high blood pressure and coronary heat disease than those of men. Schnall \& Others (1994) and Lynch (1977) have found in many studies that the relatively high incidence of heart disease in industrialized communities stems in part from the absence of positive interpersonal relationship and this lack of developing a positive interpersonal relationship with their male colleagues lead females into a depressive and frustrated state which in turn create heart disease among them in many instances.

The third hypothesis of the present study was that lower middle SES subjects would express higher stress attitudes as compared to upper middle SES subjects. The findings of the present study have provided empirical confirmation to this hypothesis. The results (Table-1) reported that regardless of type of individual and gender, lower middle SES subjects expressed significantly higher stress attitude as compared to upper middle SES (Table-4). The findings may be supported from various theoretical imports as well as empirical studies. Haynes et al. (1980) showed that development or maintenance of many health problems are frequently seen in the life style of lower middle SES individuals. 
In such a society there have been tremendous changes in the environment and conditions of living of human beings. In those changing environment of lower middle SES, incessant stimulation of the sympathetic nervous system is largely responsible for the high incidence of hypertension and other similar serious diseases. Havik et al. (1986) mentioned that in higher middle SES families, most children have regular check-ups with a family physician, are immunized against infectious diseases, and learn that preventive health measures are important. But in lower middle SES families, children generally have no regular source of medical care and rely on outpatient clinics and hospital emergency rooms, where little preventive medicine is practiced. Studies (Gentry et al. 1982) have linked coronary heart disease and high blood pressure with general environmental stress. Hypertension, heart disease, cancer were more common among those who lived in areas characterized by poverty, crime, crowding, and high divorce rates than among people who lived in areas where these kinds of stress were low. Harburg et et. al., (1973) found that the connection between environmental stress and hypertension appeared in both lower middle and upper middle SES people, but was more pronounced among lower middle SES people. Apparently genetic makeup and minority status combine with other stressors to increase rates of hypertension and coronary heart disease among lower middle SES people. Hewitt et al. (2003) found that child abuse, domestic abuse, financial stress, lack of proper education, divorce due to marital conflict are more frequent in lower middle SES families and because of these major reasons, people of these families suffer from chronic illness like cancer or CHD than people in upper middle SES families.

\section{Concluding Remarks}

In conclusion, it may be said that the study is concerned with the integration of several factors that might have both predisposing and precipitating effects on cancer and cardiac patients. People who interact closely with others are better able to avoid stress due to cancer or cardiac disease than those who remain isolated from interpersonal contract. When illness does occur, people who receive social support recover more quickly than those who do not. The utilization of social support is associated with decreased risk of heart disease among the elderly. Thus, social support reinforces the cancer \& cardiac patients sense of worth and being loved, and in such a way, acts as a protective buffer during times of crisis. Thus this study would provide an insight especially to the concerned persons and more generally to all peoples of the society on how crucial moments of life due to chronic illness could be dealt positively through social support, familial support, positive emotional regulation and self help networks.

\section{References}

Agnes END 1980. Hostility Patterns of Cancer Patients on Projective Tests. Unpublished M. Phil. dissertation. Bangalore University. Bangalore.

Allen R, Newman SP and Souhami RL. 1997. Anxiety and depression in adolescent cancer: findings in patients and parents at the time of diagnosis. European Journal of Cancer. 33(8), 1250-1255.

American Heart Association 1984. Heart Disease and Stroke Statistics-1985 Update. Dallas, Tex: American Heart Association.

Antoni MH, Lutgendorf SK and Cole SW. 2006. The influence of bio-behavioural factors on tumour biology: Pathways and mechanisms. Nature Reviews Cance. 6(3): 240-248.

Bedinghaus J, Leshan L and Diehr S. 2001. Coronary artery disease prevention: what's different for women. American Family Physician. 63: 1393-1400.

Bennett GG, Merritt MM and Sollers JJ. 2004. Stress, coping, and health outcomes among African Americans: a review of the John Henryism hypothesis. Psychol Health. 19:369-383.

Bittner V. 2006. Perspectives on dyslipidemia and coronary heart disease in women: an update. Current Opinion in Cardiology. 21: 602-607.

Byrnes DM, Antoni, MH and Goodkin K. 1998. Stressful events, pessimism, natural killer cell cytotoxicity, and cytotoxic/suppressor T cells in HIV+ Black women at risk for cervical cancer. Psychosom Med. 60:714-722.

Dahlquist LM, Czyzewski DI, and Jones CL. 1996. Parents of children with cancer: a longitudinal study of emotional distress, coping style, and marital adjustment two and twenty months after diagnosis. Journal of Pediatric Psychology. 21: 541-554.

Esterling BA, Kiecolt-Glaser JK and Glaser R. 1996. Psychosocial modulation of cytokine-induced natural killer call activity in older adults. Psychosom Med. 58:264-272.

Gentry WD, Chesney AP, Gary HE, Hall RP and Harburg E. 1982. Habitual Anger-Coping Styles: I. Effect on Mean Blood Pressure and Risk for Essential Hypertension, Psychosomatic Medicine. 44(2) :

Goldenberg LB, Nevid JS, Pelcovitz D and Carmony TM. 2002. Posttraumatic stress symptomatology in mothers of pediatric cancer survivors. Psychology and Health. 17(4): 501-511.

Goldstein MJ. 1973. Individual Differences in Response to Stress; American Journal of Community Psychology. 2: 113-37.

Harburg E, Erfurt JC, Hauensteiri LS, Chape C, Schull W and Schork MA. 1973. Socio-ecological stress, suppressed hostility, skin color, and black-white male blood pressure: Detroit. Psychosom Med. 35:276-296.

Havik OE, and Maeland JG. 1986. Dimensions of verbal denial in myocardial infarction. Scandinavian Journal of Psychology. 27: 326-339.

Haynes SG, Feinleib M and Kannel WB. 1980. The relationship of psychosocial factors to coronary heart disease in the Framingham Study: III. Eight year incidence of coronary heart disease. American Journal of Epidemiology. 111: 38-58.

Hewitt M, Rowland JH and Yanik R. 2003. Cancer survivors in the United Sates: Age, health, and disability. J Gerontol: Med Sci. 58: 82-91.

James SA and Kleinbaum DG. 1976. Socioecologic stress and hypertension related mortality rates in North Carolina. Am J Public Health. 66:354-35.

Kessler RK and Neighbors HW. 1991. A new perspective on the relationship among race, social class and psychological distress. Journal of Health and Social Behaviour. 27: 107-115. 
Lazarus RS. 1974. Psychological stress and the coping process. New York: McGraw-Hill.

Loomba RS, and Arora R. 2008. Prevention of coronary heart disease in women. Therapeutic Advances in Cardiovascular Disease. 2: 321-327.

Lynch, JJ 1977. The broken heart. New York: Basic Books.

Mosca L, Linfante AH and Benjamin EJ. 2005. National study of physician awareness and adherence to cardiovascular disease prevention guidelines in the United States. Circulation, 111: 499-510.

Pischon T, Hu FB, Rexrode KM, Girman CJ, Manson JE, and Rimm EB. 2008. Inflammation, the metabolic syndrome, and risk of coronary heart disease in women and men. Atherosclerosis. 197: 392-399.

Rahman A. 2010. Stress and Coping Attitudes of Cancer and Cardiac Patients as a function of Personality and Socio-demographic factors in Bangladesh, an unpublished Ph. D. Dissertation. Department of Psychology, Rajshahi University, Rajshahi, Bangladesh.

Reiche,EM, Nunes SO and Morimoto HK. 2004. Stress, depression, the immune system, and cancer. The Lancet Oncology. 5(10): 617-625.

Rosenman RH, Brand RJ, Jenkins CD, Friedman M, Straus R and Wurm M. 1975. Coronary disease in western collaborative group study: Final follow-up experience of $81 \frac{1}{2}$ years. Journal of the American Medical Association. 223: 872-877.

Schnall PL, Landsbergis PA and Baker D. 1994. Job strain and cardiovascular disease. Annual Review of Public Health. 15: 381-411.

Schnall PL, Pieper C, Schwartz JE, Karasek RA, Schlussel Y, Devereux RB, Ganau A, Alderman M, Warden K and Pickering TG. 1990. The relationship between “job strain,” workplace diastolic blook pressure, and left ventricular mas index. Journal of the American Medical Association 263: 1929-1972.
Selye H. 1974. Stress without Distress, New York: J.B. Lippincott, 14. Shapiro D, Goldstein IB and Jamner LD. 1995. Effects of anger and hostility, defensiveness, gender and family history of hypertension on cardiovascular reactivity. Psychophysiology. 32: 425-435.

Sher L. 1998. The role of the immune system and infection in the effects of psychological factors on the cardiovascular system. Can J Psychiatry. 43: 954-955.

Spiegel D. 1997. Psychosocial Aspects of Breast Cancer Treatment, Seminars in Oncology. 24, 36-47.

Srivastava S and Broota KD. 1987, Stress and Cancer; Journal of Personality and Clinical Studies. 3 (2): 89-94.

Tennant C. 1999. Life stress, social support and coronary artery disease. Austral N Z J Psychiatry. 33: 636-641.

Thaker PH, Han LY and Kamat AA. 2006. Chronic stress promotes tumor growth and angiogenesis in a mouse model of ovarian carcinoma. Nature Medicine. 12(8): 939-944.

Thom T, Haase N, Rosamond W. 2006. For the American Heart Association Statistics Committee and Stroke Statistics Subcommittee. Heart Disease and Stroke Statistics-2006 update. Circulation. 113: 85-151.

Williams RB, Barefoot JC, Haney TH, Harrel FE, Blumenthal J, Pryor DB and Peterson B. 1982. Type A behaviour and angiographically documented coronary atherosclerosis in a sample of 2,289 patients. Paper presented at the annual meeting of the psychosomatic society.

Manuscript received on 28 August 2012 and revised on 15 December 2012 